\title{
Development and evaluation antitumor activity of PEGylated liposomal doxorubicin on tumor-bearing BALB/c-Foxnlnu mice model
}

\author{
Hue Pham Thi Minh ${ }^{1}$, Linh Le Phuong ${ }^{1}$, Hai Nguyen Thanh ${ }^{2}$, Son Ho Anh ${ }^{3}$, Tung Bui Thanh ${ }^{2 *}$ \\ ${ }^{1}$ Hanoi University of Pharmacy, 15 Le Thanh Tong, Hoan Kiem, Ha Noi, Vietnam. ${ }^{2}$ School of Medicine and Pharmacy, Vietnam National University, \\ Hanoi, 144 Xuan Thuy, Cau Giay, Ha Noi, Vietnam. ${ }^{3}$ Vietnam Military Medical University, 160 Phung Hung, Ha Đong, Ha Noi, Vietnam.
}

\begin{tabular}{|c|c|}
\hline ARTICLE INFO & ABSTRACT \\
\hline Article history: & \multirow{9}{*}{$\begin{array}{l}\text { Doxorubicin hydrochloride is an antitumor antibiotic derived from anthracyclines. It has had limited use because } \\
\text { of its dose-related cardiotoxicity and myelosuppression. Liposomes have been used as a vehicle for } \\
\text { administration of pharmaceutical drugs because of their ability to improve the delivery of drugs to tumors, } \\
\text { increase therapeutic efficacy, and decrease toxicity to normal cells. The aim of this study is to prepare a new } \\
\text { liposomal dxorubicin on a large-scale and evaluate its antitumor activity in vivo. Liposomes were formed using } \\
\text { the hydration of a thin lipid film method, and doxorubicin was loaded through a pH gradient technique. Based on } \\
\text { TEM images, large lamellar vesicles (LUV) were formed, with sizes of } 95 \pm 10 \mathrm{~nm} \text {, having a polydispersity } \\
\text { index of } 0.138 \pm 0.02 \text { and zeta potentials of about }-27.8 \pm 2.15 \mathrm{mV} \text {. The entrapment efficiency was } \\
\text { approximately } 97 \% \text {. The therapeutic activity of PEGylated liposomal doxorubicin formulations was studied on } \\
\text { human colorectal carcinoma HT } 29 \text { tumor-bearing BALB/c-Foxn }{ }^{\mathrm{nu}} \text { mice models. Our results have shown that } \\
\text { liposome preparation can reduce the tumor volume and increase the survival rate and survival time as compared } \\
\text { with Lipo Dox. PEGylated liposomal doxorubicin demonstrated much stronger antitumor activities, and } \\
\text { statistical differences were significant when compared with free doxorubicin. }\end{array}$} \\
\hline Received on: $11 / 07 / 2015$ & \\
\hline Revised on: $04 / 08 / 2015$ & \\
\hline on: $26 / 08 / 2015$ & \\
\hline Available online: $27 / 09 / 2015$ & \\
\hline Key words: & \\
\hline Doxorubicin, liposome, & \\
\hline $\begin{array}{l}\text { PEGylated, tumor-bearing } \\
\text { mice, HT29. }\end{array}$ & \\
\hline & \\
\hline
\end{tabular}

\section{INTRODUCTION}

Doxorubicin hydrochloride (Dox) is an antitumor antibiotic derived from anthracyclines. Dox is limited using because of its dose-related cardiotoxicity and myelosuppression. The using liposomal Dox in ovary, lung, and breast cancer therapies has been encouraged due to its superior efficacy and minimum cardiotoxicity. The mechanism action of this class drug is interacted with deoxyribonucleic acid (DNA) in a variety of different ways, including intercalation (squeezing between the base pairs), DNA strand breakage, and inhibition of activity of topoisomerase II. The liposomal forms has advantages that it allow Dox to remain longer time in the circulation system, and delivery of a larger amount of the drug to target cancerous cells

\footnotetext{
* Corresponding Author

Tung Bui Thanh, School of Medicine and Pharmacy, Vietnam National University, Hanoi, Floor 5 Building Y1, 144 Xuan Thuy, Cau Giay, Ha Noi, Vietnam,e-mail: tungasia82@yahoo.es

Tel: +84-4-85876172; Fax: +84-0437450188
}

or tumors, avoid the normal cell, increasing the bioavailability, decreasing the metabolism, and increasing Dox's efficaz therapeutic (Gabizon, Shmeeda et al.,2003, Jiang, Lionberger et al.,2011, Barenholz 2012). When the drug is prepared in liposomal form, its therapeutic effects of anti-cancer drugs could be increased and the toxic side effects decreased. However, the conventional liposome has limited effectiveness because of their rapid uptake by the cells of the reticuloendothelial system (RES), reducing the amount of the drug that reaches the tumor.

To overcome this limitation, by covalently attaching polyethylene glycol (PEG) to the lipid bilayers, smaller and more rigid liposomes are produced. Pegylated liposomal Dox is a longcirculating formulation of liposomal Dox. It was approved for using by United States Food and Drug Administration in 1995 and this has opened a new breakthrough of nanotechnology in drugdelivery systems to penetrate target cells to deliver the bioactive agent (Barenholz 2012). Pegylated liposomal Dox injection has attracted a lot of attention of many scientists around the world (Gabizon, Shmeeda et al.,2003). 
PEG forms a protective layer over the liposome surface and PEGylated liposomal has long circulation time and provides slow release of an encapsulated drug (Harris and Chess 2003). Therefore, PEG coated liposomes can reduce the uptake by the cells of the RES and have a longer circulation time, consequently, results in an increased accumulation in tumors (Gabizon and Martin, 1997).

In the previous our study, we have prepared the PEGylated liposomal Dox and evaluated its effect of cytotoxicity on two cell lines A549 and HT29 and provided promise results (Linh et al., 2015). Therefore in this study, we focused on prepare PEGylated liposomal Dox injection at large-scale and evaluate in vivo the effect on human colorectal carcinoma HT-29 tumorbearing animal model

\section{MATERIAL AND METHODS}

\section{Reagent and instruments \\ Reagent}

Doxorubicin hydrochloride, hydrogenated soybean phosphatidylcholine (HSPC) (Lipoid), (1,2-distearoyl-sn-glycero3-phosphoethanolamine-N-[amino (polyethylene glycol) -2000] (ammonium salt) (DSPE-(PEG) $\left.2000-\left(\mathrm{NH}_{2}\right)\right)$, Hepes (N-2hydroxyethylpiperazine-N'-2-ethanesulfonic acid), cholesterol, chloroform, sodium hydroxide, ammonium sulfate, potassium dihydrogen phosphate, disodium hydrogen phosphate, phosphoric acid, triton X 100 (octyl phenol ethoxylate), 3-[4,5-dimehyl-2thiazolyl]-2,5-diphenyl-2H-tetrazolium bromide (MTT). All other reagents and solvents used to meet requirements for pharmaceutical and analytical grade. Reference drugs: Solution Dox Ebewe for injection $25 \mathrm{ml}$ vials, $2 \mathrm{mg} / \mathrm{ml}$ (Ebewe Pharma ) and Lipo Dox Injections (TTY Bio pharm).

\section{Instruments}

The evaporation system Rovapor R-210; Spectra/Por ${ }^{\circledR} 4$ Dialysis Membrane, MWCO: 12,000-14,000 Daltons; Analyzer size system Zetasizer ZS90; Ultrasound Machines; UV-VIS Spectrophotometer; pH InoLab meter; Tangential Flow MicroKros Filter Modules ${ }^{\circledR}$ (Spectrum Labs) with membrane polysulfone $10 \mathrm{kD}, 28 \mathrm{~cm}^{2}$ (USP), Centrifuge Hettich Universal 320R (Germany), High Pressure Homogenizers EmulsiFlex-c5 (AvestinCanada), magnetic stirrer and other common tools.

\section{Methods}

\section{Preparation of the PEGylated liposomal Dox injection:}

Using the method of hydration of a thin lipid film: Bangham method.

Weight and dissolve phospholipids: HSPC, cholesterol và $D_{S P E}-P_{2000}(3: 1: 1 \mathrm{w} / \mathrm{w})$ in chloroform. Then, the organic solvent was removed by evaporation using the Rovapor R-210 system at $50^{\circ} \mathrm{C}$ for $12 \mathrm{~h}$ to form the dry lipidic film on the flask wall. Hydrate the thin lipid film by adding a buffer citrate solution $\mathrm{pH} 4.0$ at $60^{\circ} \mathrm{C}$ for $2 \mathrm{~h}$.
- Reduce liposome size by using High Pressure Homogenizers EmulsiFlex with nitrogen gases. Compress 10 cycles at pressure 10,000 psi, maintaining temperature at $60^{\circ} \mathrm{C}$. Filter suspension through membrane filter $0.2 \mu \mathrm{m}$.

- Change the external buffer environment of liposome with buffer Hespes pH 7.5 using tangential flow filtration. Weight exactly an amount of doxorubicin hydrochloride, add to the suspension of liposome, stir with $80 \mathrm{rpm}$ for $30 \mathrm{~min}$, temperature maintaining at $50 \pm 2{ }^{\circ} \mathrm{C}$.

- $\quad$ Filter the product through membrane filter $0.2 \mu \mathrm{m}$ and then packed into $10 \mathrm{ml}$ glass closed with rubber and aluminum cap, keep in a refrigerator from $8-10{ }^{\circ} \mathrm{C}$.

\section{Liposome evaluation \\ Morphology and structure of liposome}

Using the method of negative staining transmission electron microscopy (TEM)

\section{Liposome size, distribution and Zeta potential}

Using the method of dynamic light scattering (DLS) with instrument Zetasizer ZS90. Dilute suspension of liposome 200 times with deionized water.

Quantification of Dox: using a HPLC method.

Mobil phase

Dissolve $1 \mathrm{~g}$ of sodium lauryl sulfate in $1000 \mathrm{~mL}$ mixture of water-acetonitril-methanol-phosphoric acid (400:450: 150:2), adjust to $\mathrm{pH} 3.6 \pm 0.1$ by solution sodium hydroxyde $2 \mathrm{~N}$.

Detector: UV -VIS, $254 \mathrm{~nm}$.

Flow rate: $1.2 \mathrm{ml} / \mathrm{min}$

Injection volume: $20 \mu \mathrm{L}$

\section{Entrapment efficiency}

Add $1 \mathrm{~mL}$ of PEGylated liposomal Dox suspension into dialysis bag and hang the bag in an Erlenmeyer flask containing $100 \mathrm{ml}$ of phosphate buffer $\mathrm{pH}$ 4.0. Maintain system at temperature $8-10{ }^{\circ} \mathrm{C}$ for 12 hours. Take the solution from outside of the dialysis bag and measure optical density at $233 \mathrm{~nm}$ wavelength. calculated by:

The percentage entrapment efficiency of the drug was

$$
\% \text { Entrapment efficiency }=\frac{\mathrm{mo}-\mathrm{m}}{\mathrm{mo}} \times 100 \%
$$

m, mo: amount of Dox diffused through the dialysis membrane and amount of Dox initial.

\section{Quantification of phospholipid}

Using a spectrophotometric method with wavelength 475 $\mathrm{nm}$. The ratio of moles of Dox/phospholipids is calculated based on the results of quantification of phospholipids and Dox. 


\section{Evaluation in vivo effects of PEGylated liposomal Dox on tumor implantation in mice Cell lines}

The HT 29 colon cancer cell line was purchased from company ATCC, USA. Cells were grown in RPMI medium (ATCC, USA) containing $10 \%$ Fetal Bovine Serum and 1\% streptomycin-penicillin.

\section{Animals}

6 weeks old BALB/c-Foxn $1^{\text {nu }}$ mice that were purchased from Charly-River company (USA). All animal experiments were performed in accordance with the guidelines of Vietnam Military Medical University. Mice were kept under pathogen-free conditions, under a $12 \mathrm{~h}$ light/dark cycle, controlled temperature $\left(28 \pm 0,5^{\circ} \mathrm{C}\right)$ and humidity $55 \pm 5 \%$. All animals were maintained accordingly to a protocol approved by the Ethical Committee of the Vietnam Military Medical University and following the international rules for animal research. They were fed ad libitum (Zeigler, USA) with a standard diet be sterilized before use. Mice were randomly maintained five animals per group. The cages were located in the system with good ventilation and filter membrane to ensure the free of pathogens.

\section{Tumor Implantation, Treatment and Evaluation}

HT-29 cells used for xenograft tumors were prepared by trypsinization. The cells were washed and re-suspended at a concentration of $10^{7}$ cells $/ \mathrm{mL}$ in PBS, which was then inoculated $(0.1 \mathrm{ml} /$ mouse) subcutaneously (s. c.) into the right thigh of the mice. This process is done in sterile conditions.

Tumors were monitored 2 times per week to track the developments at site of injection (right thigh) by observation, touch and tumor volume was measured by NSK Micrometer accurate. After tumor implantation has the size about $10 \mathrm{~mm}$ in diameter (about 3 weeks after cells were injected), mice were randomly divided in four groups: Control group (injected physiological saline solution); Dox (injected solution Dox Ebewe); Lipo Dox (injected solution of Lipo Dox Injections); and Liposome (injected our PEGylated liposomal Dox prepared). Mice were injected intravenously in tails vein with corresponding doses of Dox $5 \mathrm{mg} / \mathrm{kg} /$ body weight. Drug treatment was done by 1 time/week during 3 weeks. All changes in tumor volume, body weight, mice's dead time were noted.
Measuring process of tumor volume was stopped when there is mouse died at any group. The process of tracking the ratio of alive and death mice was stopped when all mice in Control group died (Fig. 1). The survival rate was studied by Kaplan-Meier analysis. Tumor volume was calculated using the formula:

$$
\mathrm{V}=0.5 \times \mathrm{D} \times \mathrm{R}^{2}
$$

V: Tumor volume; D: Tumor length; R: Tumor width

\section{Statistical analysis}

All data are shown as the mean \pm standard deviation (SD). One-way analysis of variance (ANOVA) was used to determine significance among groups. Statistical significance was set at $\mathrm{p}<0.05$

\section{RESULTS AND DISCUSSION}

\section{Preparation and characterization of PEGylated liposomal Dox at scale $100 \mathrm{vial} / \mathrm{batch}(1000 \mathrm{ml} / \mathrm{batch})$}

We have prepared three batches of PEGylated liposomal Dox at scale $100 \mathrm{vial} / \mathrm{batch}(1000 \mathrm{ml} / \mathrm{batch})$ and analyzed some properties of the obtained PEGylated liposomal Dox including observation, $\mathrm{pH}$, mean particle size, polydispersity index (PDI), zeta potential, Dox content total $(\mathrm{mg} / \mathrm{ml})$, drug entrapment efficiency, and Dox/phospholipid ratio. The results were shown in Table 1 and Fig. 2.

Table 1: Characterization of PEGylated liposomal Dox $2 \mathrm{mg} / \mathrm{ml}$.

\begin{tabular}{ll}
\hline Parameter & Results \\
\hline Observation & Suspension, color orange-red \\
PH & $7.2 \pm 0.2$ \\
Volume vials $(\mathrm{ml})$ & $10.0+0.5$ \\
Mean particle size (diameter, $\mathrm{nm})$ & $95 \pm 10$ \\
Polydispersity index $-\mathrm{PDI}$ & $0.138 \pm 0.02$ \\
Zeta potential $(\mathrm{mV})$ & $-27.8 \pm 2.15$ \\
Dox content total $(\mathrm{mg} / \mathrm{ml})$ & $2.053 \pm 0.009$ \\
Drug entrapment efficiency $(\%)$ & $97.5 \pm 2.3$ \\
Dox/phospholipid ratio $(\mu \mathrm{g} / \mu \mathrm{mol})$ & $138.41 \pm 0.023$ \\
\hline
\end{tabular}

We performed the determination of particle size to confirm the desired liposome size range. The size of particles plays important role due to their interaction with the biological environment. When particles are loaded by intravenous administration, their ability to pass or leave the vascular capillaries effectively is dependent on the size (Gauger et al.,2001).

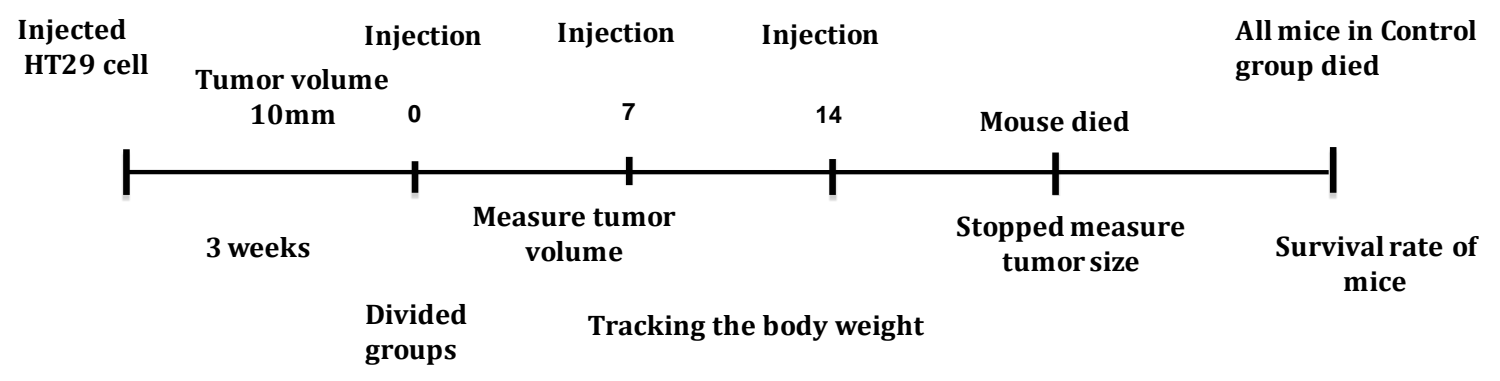

Fig. 1. Chronological scheme of procedures with mice groups. 
Referring to Table 1, PEGylated liposomal Dox has a size of $95 \pm 10 \mathrm{~nm}$. That means our liposome with small particle size $(<200 \mathrm{~nm})$ could increase the accumulation of drug in the tumor by augmented permeability and retention effect.

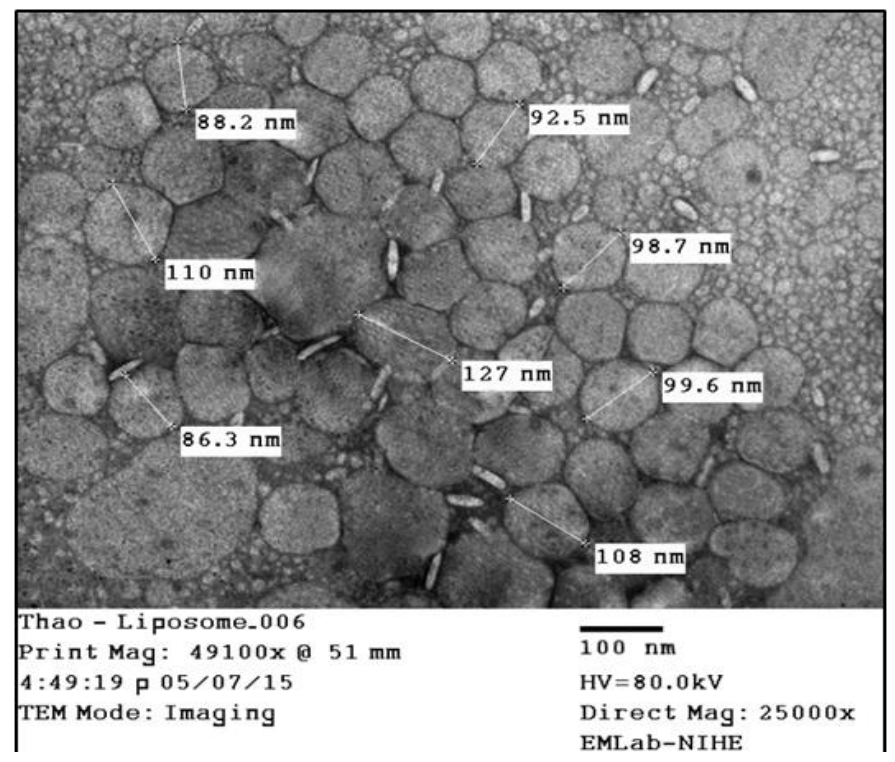

Fig. 2: PEGylated liposomal Dox was taken by TEM.

The polydispersity index value is a measure of the heterogeneity of particle sizes in a compound. Liposomes with PDI value between 0.1 and 0.25 display more uniformity and physical stability. In case of PDI value more than 0.5 indicates the poor uniformity of mixture (Pereira-Lachataignerais, Pons et al., 2006). Our PDI values of liposomes are $0.138 \pm 0.02$ which confirm the uniformity and homogeneity of our PEGylated liposomal Dox.

The value of zeta potential (ZP) confirms the stability of the systems. It presents the repulsive forces between the particles. Particles having a ZP of less than $-25 \mathrm{mV}$ or more than $+25 \mathrm{mV}$ are usually considered stable. Our PEGylated liposomal Dox preparations have zeta potential values $-27.8 \pm 2.15 \mathrm{mV}$ (Table 1), then they are considered stable.

As showed in Table 1, our liposomes contained very high entrapment efficiency, $97.5 \%$. That confirmed that our liposome preparations have meet requirements of a liposome product.

\section{Liposomes Formation and Morphology}

TEM images in Fig. 2 demonstrate the formation of liposomes. According to the TEM images, particle PEGylated liposomal Dox have fairly evenly sized, with average size from 80-130 $\mathrm{nm}$ and single layer.

\section{Evaluation in vivo the effect of PEGylated liposomal Dox on human colorectal carcinoma HT-29 tumor-bearing BALB/c- Foxn1 $^{\text {nu }}$ mice model}

The in vivo tumor growth curve was presented in Fig. 3. There is no difference significantly between tumor volume of all groups in the early days. However, until the day $9^{\text {th }}$, tumor volume of group Lipo Dox and group PEGylated liposomal Dox is differences statistical significantly with its Control and Dox group. The tumor volume of group Lipo Dox and group PEGylated liposomal Dox are very similar and they are only a half of its Control group. There is no difference significantly between in tumor volume of Dox group and Control group.

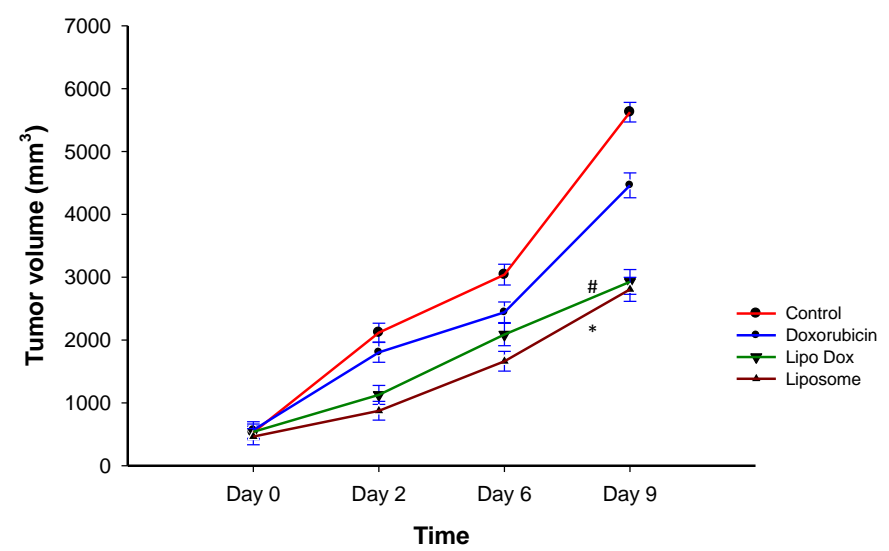

Fig. 3: In vivo growth curve of HT-29 tumor cells. Results are expressed as the mean \pm SD $(n=5)$. ("Significantly different between Lipo Dox group and Control group $(\mathrm{p}<0.05)$.

We also determined the activity of suppression tumor growth of our prepared PEGylated liposomal Dox. Our PEGylated liposomal Dox have been showed strong antitumor activity on human colorectal carcinoma HT-29 tumor-bearing BALB/cFoxn1nu mice model as showed in Fig. 4. The capacity in delayed tumor growth HT-29 cells of our PEGylated liposomal Dox is notable, even stronger then reference Lipo Dox. Percentage of tumor growth inhibition of our PEGylated liposomal Dox is over then $50 \%$, stronger more than 2.5 times then free Dox. The solution of free Dox has weak activity, compared with Control group is not differences significantly $(p>0.05)$.

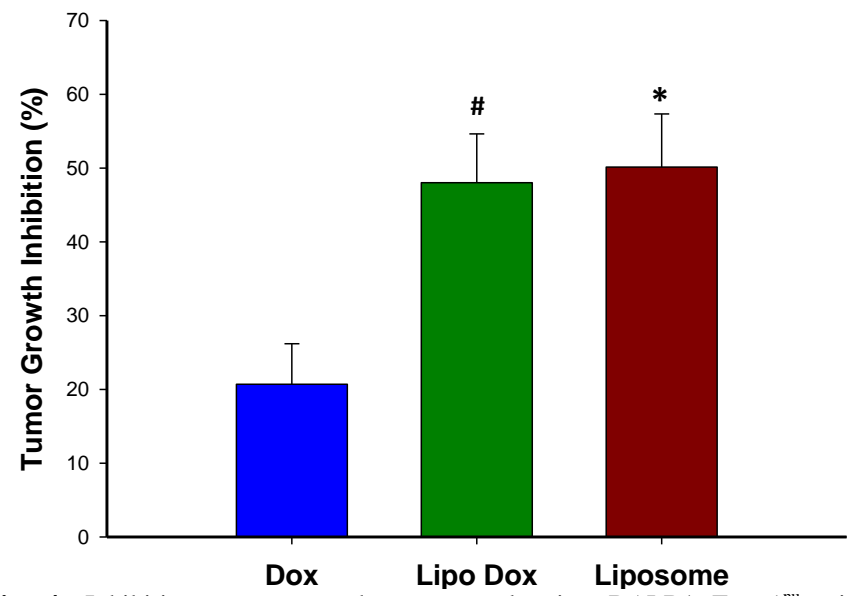

Fig. 4: Inhibition tumor growth on tumor-bearing $B A L B / c-F o x n 1^{\text {nu }}$ mice model ("Significantly different between Lipo Dox group and Control group $(\mathrm{p}<0.05)$. Results are expressed as the mean \pm SD $(n=5)$. " Significantly different between our Liposome group and Control group $(\mathrm{p}<0.05)$.

\section{Mice body weight}

In the all mice group, there are no changes in mice's body weight during the treatment. The mice's body weight in the 
treatment groups with our PEGylated liposomal Dox injection, reference Lipo-Dox and free DOX compared with Control group did not differ significantly before and after treatment. There is also no difference between all groups at the same time of measurement. This shows that doses of $5 \mathrm{mg} / \mathrm{kg}$ of all formulations in the study are safely and do not affect to mice's body weight.

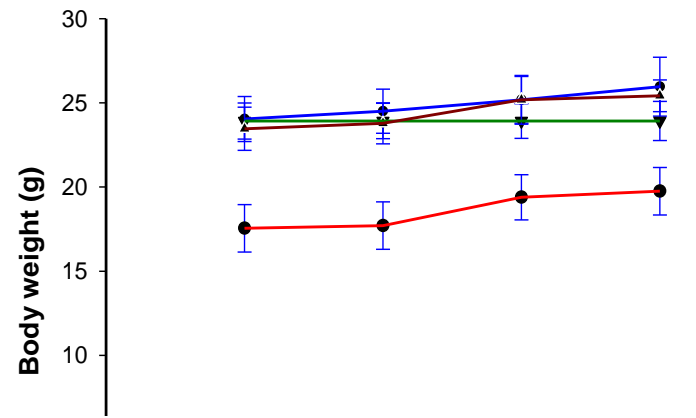

Fig. 5: Mice body weight change during the treatment (g). Results are expressed as the mean $\pm \operatorname{SD}(n=5)$.

\section{Survival rate and average survival time of animals}

We compared the survival rates of on human colorectal carcinoma HT-29 tumor-bearing mice following the four different treatment regimens. The survival rates is presented in Fig. 6.

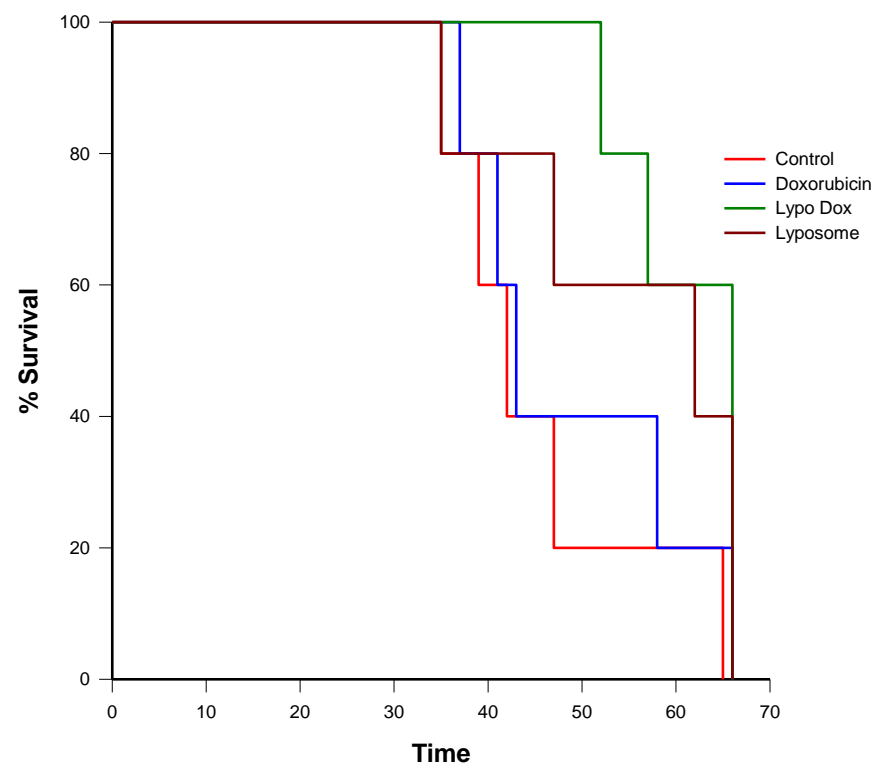

Fig. 6: Liposomal Dox enhanced survival. The Kaplan-Meier survival curve shows improvement of life span of tumor-bearing mice treated with Lipo Dox and Liposomal Dox. Results are expressed as the mean $\pm \mathrm{SD}(\mathrm{n}=5)$.

At the end of experiments (when all mice in Control group died), survival mice in with Lipo Dox and our Liposomal Dox remain $60 \%$ of total animals, and in free Dox is only $20 \%$. It can be explained that our Liposomal Dox is less toxic than free Dox. This result was confirmed in the survival time which is presented in Fig. 7. As shown in Fig. 7, survival times for the four different groups were 45.6 days (Control group), 49 days (free Dox), 61.4 days (Lipo Dox) and 55.2 days (our PEGylated liposomal Dox), respectively. Thus, our PEGylated liposomal Dox treatment increased medium survival time by $21.0 \%$ compared to the saline control group, by $12.6 \%$ compared to the free DOX group. These experiments demonstrated that the administration of our PEGylated liposomal Dox in 3 doses during two-week period not only afforded better inhibition of tumor growth but also improved the survival of human colorectal carcinoma HT-29 tumor-bearing BALB/c-Foxn $1^{\mathrm{nu}}$.

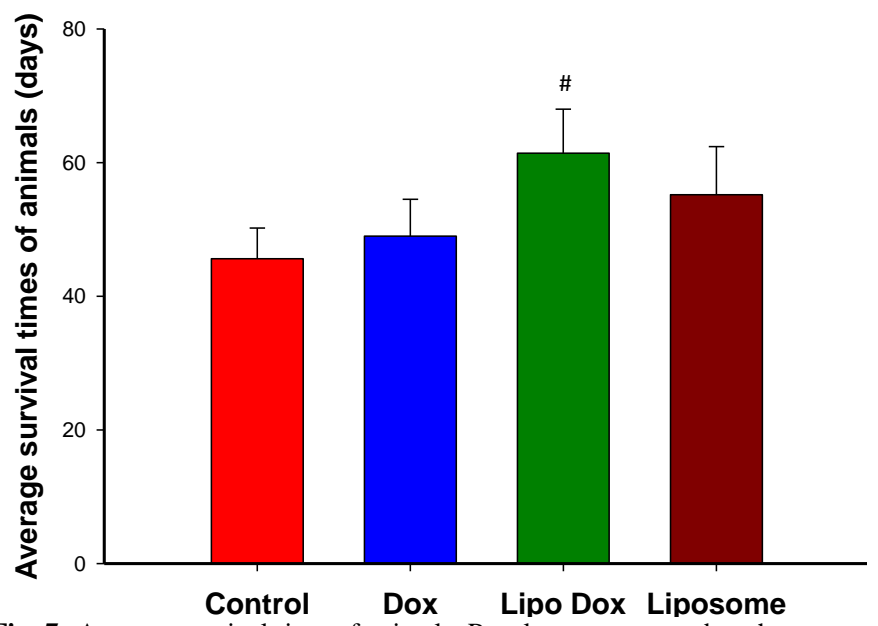

Fig. 7: Average survival time of animals. Results are expressed as the mean \pm $\mathrm{SD}(\mathrm{n}=5)$.

Our results indicated PEGylated liposomal Dox could be used as a tumor-targeting and tumor-penetrating ligand for tumor targeting drug delivery systems. Our result was agreed with previous reports (Ogawara et al.,2009; Yu et al., 2012). Lin et al., have shown the nanoliposomal Dox could deliver Dox for treatment of human colorectal carcinoma HT-29 tumor-bearing mice with greater therapeutic efficacy as suppression of tumor growth and extended survival in contrast to the free drug. It also reported that a lower Dox uptake in the principal sites of toxicity of the free drug, such as heart and skin, and reduced myelosuppression and diminished cardiotoxicity ( $\mathrm{Yu}$ et al.,2012). Ogawara et al., have shown that the significant extension of the mean survival time after the treatment with PEG liposomal doxorubicin in the Dox-resistant colon-26 cancer cells-bearing mice model in a dose-dependent manner. These authors suggested the anti-tumor effect of PEGylated liposomal Dox on human colorectal carcinoma HT-29 tumor-bearing mice could be explained by its cytotoxic effect of Dox on vascular endothelial cells in the tumor (Ogawara et al., 2009). In our previous study, we have shown after 48 hours, our PEGylated liposomal Dox exerted its weak cytotoxicity and after 72 hours it it was most effective with IC50 $0.86 \mu \mathrm{g} / \mathrm{ml}$ on HT 29 cells (Linh et al.,2015). This data was confirmed again in vivo antitumor activity on human colorectal carcinoma HT-29 tumor-bearing BALB/c-Foxn $1^{\text {nu }}$ mice model. 


\section{CONCLUSION}

In this study, the PEGylated liposomal Dox was prepared successfully at large-scale $1000 \mathrm{mg} / \mathrm{batch}$ (100 vials/batch). Liposomal formulations have particle size, distribution, zeta potential in the acceptable range and high drug entrapment efficiency (over 97\%).

Our PEGylated liposomal Dox has survival rate 3 times and extends survival time more than free Dox on human colorectal carcinoma HT-29 tumor-bearing BALB/c-Foxn $1^{\text {nu }}$ mice model. PEGylated liposomal Dox can reduce tumor volume and have less toxic, similar than reference Lipo Dox and difference significantly with free Dox.

\section{CONFLICT OF INTERESTS}

The authors declare that there is no conflict of interest regarding the publication of this paper.

\section{REFERENCES}

Barenholz, Y. Doxil(R)--the first FDA-approved nano-drug: lessons learned. J Control Release, 2012; 160(2): 117-134.

Gabizon, A. and F. Martin. Polyethylene glycol-coated (pegylated) liposomal doxorubicin. Rationale for use in solid tumours. Drugs, 1997; 54 (Suppl 4): 15-21.

Gabizon, A., H. Shmeeda and Y. Barenholz Pharmacokinetics of pegylated liposomal Doxorubicin: review of animal and human studies. Clin Pharmacokinet, 2003; 42(5): 419-436.
Harris, J. M. and R. B. Chess. Effect of pegylation on pharmaceuticals. Nat Rev Drug Discov, 2003; 2(3): 214-221.

Heldt, N., M. Gauger, J. Zhao, G. Slack, J. Pietryka and Y. Li. Characterization of a polymer-stabilized liposome system." Reactive and Functional Polymers, 2001; 48(1-3): 181-191.

Hue, P. T. M., L. P. Linh, N. V. Lam, N. T. Hai, H. A. Son, N. L. Toan and B. T. Tung. Developing and evaluating in vitro effect of pegylated liposomal doxorubicin on human cancer cells. Journal of Chemical and Pharmaceutical Research, 2015; 7(3): 2239-2243.

Jiang, W., R. Lionberger and L. X. Yu. In vitro and in vivo characterizations of PEGylated liposomal doxorubicin. Bioanalysis, 2011; 3(3): 333-344.

Lin, J., Y. Yu, S. Shigdar, D. Z. Fang, J. R. Du, M. Q. Wei, A. Danks, K. Liu and W. Duan. Enhanced antitumor efficacy and reduced systemic toxicity of sulfatide-containing nanoliposomal doxorubicin in a xenograft model of colorectal cancer. PLoS One, 2012; 7(11): e49277.

Ogawara, K., K. Un, K. Tanaka, K. Higaki and T. Kimura. In vivo anti-tumor effect of PEG liposomal doxorubicin (DOX) in DOXresistant tumor-bearing mice: Involvement of cytotoxic effect on vascular endothelial cells. J Control Release, 2009; 133(1): 4-10.

Pereira-Lachataignerais, J., R. Pons, P. Panizza, L. Courbin, J. Rouch and O. Lopez. Study and formation of vesicle systems with low polydispersity index by ultrasound method. Chem Phys Lipids, 2006; 140(1-2): 88-97.

\section{How to cite this article:}

Hue Pham Thi Minh, Linh Le Phuong, Hai Nguyen Thanh, Son Ho Anh, Tung Bui Thanh. Development and evaluation antitumor activity of PEGylated liposomal doxorubicin on tumor-bearing BALB/c-Foxn1nu mice model. J App Pharm Sci, 2015; 5 (09): 001-006. 